\title{
Comparação do ultrassom pulsado e contínuo no reparo tendíneo de ratos
}

\author{
Comparison of pulsed and continuous ultrasound on tendon repair in rats
}

Gladson Ricardo Flor Bertolini', Taciane Stein da Silva², Adriano Polican Ciena ${ }^{3}$, Elisângela Lourdes Artifon ${ }^{4}$

RESUMO I No tratamento de lesões tendíneas, o uso do ultrassom surge como possibilidade terapêutica, apesar de lacunas sobre seus efeitos clínicos. O objetivo foi avaliar dois protocolos de ultrassom terapêutico sobre dor e edema após trauma tendíneo. Vinte e um ratos Wistar foram submetidos a trauma no tendão calcâneo e divididos em três grupos: sham (GS); ultrassom contínuo (GUC); e ultrassom pulsado (GUP). O trauma ocorreu sobre a face lateral do tendão calcâneo direito, com energia de $0,40 \mathrm{~J}$. A dor foi avaliada pelo teste de incapacidade funcional e o edema, pelo diâmetro laterolateral. Foram realizadas avaliações previamente à lesão; após 1 hora da indução da lesão; após o 10 tratamento; 2, 8 e 24 horas após lesão; e após o 50 dia. O tratamento ocorreu em 5 dias, com transdutor de $1 \mathrm{MHz}$, durante 3 minutos, sobre o local do trauma, com dose de 0,4 W/cm² SATA. Os resultados da incapacidade funcional para GS mostraram aumento da nocicepção. Para GUC houve aumento ao comparar a avaliação 1 (AV1) com as avaliações 2 (AV2), 3 (AV3) e 4 (AV4); ao comparar AV2 com as avaliações 5 (AV5) e 6 (AV6) houve diminuição de valores. Para GUP houve aumento ao comparar AV1 com AV2 e AV3, mas ao comparar AV2 com as seguintes, houve diminuição significativa a partir de AV4. Para o edema, os grupos tratados produziram aumento inicial, com redução nas últimas avaliações. O ultrassom terapêutico produziu diminuição de dor e edema, mais precocemente para a forma pulsada.

Descritores | tendão do calcâneo; ultrassom; dor; regeneração.
ABSTRACT I In tendon injuries treatment, the use of ultrasound appears as a therapeutic option, despite the lack of their clinical effects. The aim was to evaluate two therapeutic ultrasound protocols, continuous or pulsed, on the pain and swelling after tendinous trauma. Twenty-one Wistar rats were subjected to traumatic injury in the tendon and divided in three groups: sham (GS), continuous ultrasound (GUC), and pulsed ultrasound (GUP). The injury occurred on right Achilles tendon lateral aspect, with energy of $\mathrm{O} .40 \mathrm{~J}$. The pain was assessed by the functional disability test and edema by latero-lateral diameter. The evaluations were performed before the injury; after 1 hour of the lesion induction; after the 1st treatment; 2, 8 and 24 hours after injury; and after the 5 th day. The treatment was made for 5 consecutive days, with transductor of $1 \mathrm{MHz}$, for 3 minutes on the trauma site, with a dose of $0.4 \mathrm{~W} / \mathrm{cm}^{2}$ SATA. The results of functional disability for GS showed increased nociception. For GUC, there was increased when comparing evaluation 1 (EV1) with evaluations 2 (EV2), 3 (EV3) and 4 (EV4); comparing EV2 to evaluations 5 (AV5) and 6 (AV6), there was decreased values. To GUP there was increased when comparing EV1 with EV2 and EV3, but when comparing EV2 with the following, there was a significant decrease starting from EV4. Edema, treatment groups produced an initial increase with a reduction in recent evaluations. The therapeutic ultrasound produced pain and edema reduction in rats with tendon trauma, and early in the pulsed.

Keywords I Achilles tendon; ultrasonics; pain; regeneration.

Estudo desenvolvido no Laboratório de Estudo das Lesões e Recursos Fisioterapêuticos da Universidade Estadual do Oeste do Paraná (UNIOESTE) - Cascavel (PR), Brasil.

'Professor Adjunto da UNIOESTE - Cascavel (PR), Brasil.

²Doutoranda em Farmacologia pela Universidade Federal de Santa Catarina (UFSC) - Florianópolis (SC), Brasil.

${ }^{3}$ Doutorando em Ciências pela Universidade de São Paulo (USP) - São Paulo (SP), Brasil.

${ }^{4}$ Fisioterapeuta pela UNIOESTE - Cascavel (PR), Brasil. 


\section{INTRODUÇÃO}

Os tendões têm propriedades biomecânicas altamente adaptáveis baseadas em remodelamento, por lise e síntese $^{1}$. As tendinopatias ocorrem devido à fadiga ou microtraumas à matriz. Danos aos tendões, provavelmente, ocorrem continuamente, como resultados de atividades diárias. Contudo, as células podem reparar o dano, retornando o tendão ao estado prévio ${ }^{2}$.

Forças de fricção e cargas repetitivas direcionam a lesões crônicas, que resultam em inflamação, degeneração e ruptura ${ }^{3}$. Segundo Aspenberg ${ }^{4}$, apesar de células inflamatórias se apresentarem moderadamente aumentadas em estágios tardios, presume-se que reações inflamatórias ocorram no desenvolvimento da lesão. As lesões por sobrecarga tendíneas são responsáveis por 30 a $50 \%$ de todas as lesões esportivas 5 .

Para o tratamento de tendinopatias, na fase aguda, são utilizados anti-inflamatórios não hormonais que previnem a liberação de mediadores químicos. $\mathrm{Na}$ lesão crônica, eles também são usados para ajudar a controlar a dor e a inflamação. Contudo, apresentam diversos efeitos colaterais ${ }^{1}$. Além disso, Marsolais, Côté e Frenette ${ }^{6}$, avaliando o uso do diclofenaco em modelo experimental de tendinite, observaram diminuição do edema e de células inflamatórias, mas sem melhora funcional ou biomecânica. Outra possibilidade são os corticosteroides; contudo, apresentam mais efeitos colaterais, como diminuição da resistência tendínea, da produção de colágeno e da substância basal ${ }^{1}$.

Assim outras formas terapêuticas têm espaço em casos de tendinopatias, como ultrassom e fonoforese $\mathrm{e}^{7,8}$, iontoforese, campos eletromagnéticos, crioterapia, eletroestimulação com corrente alternada, corrente galvânica, compressão intermitente, massagem profunda e termoterapia ${ }^{1}$. Especificamente para o ultrassom, a literatura apresenta efeitos benéficos com relação à aceleração do reparo tendíneo ${ }^{9-12}$. Contudo, Warden e McMeeken ${ }^{13}$ salientam uma lacuna de evidências científicas sobre os efeitos terapêuticos, bem como uma falta de padronização dosimétrica, existindo dúvidas com respeito à melhor forma de entregá-lo. Há relatos favoráveis apenas à forma pulsada ${ }^{9,12}$, contudo, outros estudos apontam melhora no reparo tendíneo também na forma contínua ${ }^{10,11}$. Assim, o objetivo deste estudo foi avaliar dois protocolos de ultrassom terapêutico variando a forma de aplicação, contínua ou pulsada, sobre a dor e o edema advindos de um trauma tendíneo.

\section{METODOLOGIA}

\section{Grupos experimentais}

Foram utilizados 21 ratos Wistar, machos, com massa de 405,60 $\pm 55,98 \mathrm{~g}$, mantidos em fotoperíodo de 12 horas, com água e ração ad libitum. Os animais foram divididos em três grupos:

- GS (n=7) - lesão traumática em tendão calcâneo direito e tratamento placebo (sham);

- GUC (n=7) - lesão traumática e tratado com ultrassom terapêutico contínuo;

- GUP (n=7) - lesão traumática e tratado com ultrassom terapêutico pulsado.

O projeto foi conduzido segundo as normas de ética em experimentação animal, sendo aprovado pelo Comitê de Ética na Experimentação Animal e Aulas Práticas da Universidade Estadual do Oeste do Paraná (UNIOESTE), sob Parecer no 0509.

\section{Modelo experimental de trauma no tendão calcâneo}

Os animais foram anestesiados com quetamina $(95 \mathrm{mg} / \mathrm{kg})$ e xilazina $(12 \mathrm{mg} / \mathrm{kg})$ intraperitonealmente. Posteriormente, foram posicionados em decúbito lateral esquerdo, expondo a região lateral do tendão calcâneo direito ao trauma. Para a produção da lesão traumática, foi utilizado um equipamento projetado pelo departamento de Engenharia Civil da UNIOESTE. Tal equipamento é constituído por uma massa de $575 \mathrm{~g}$, e partiu de uma mesma altura inicial $(7 \mathrm{~cm})$, em queda sobre a face lateral do tendão calcâneo direito de cada animal, perfazendo uma energia de impacto de aproximadamente $0,40 \mathrm{~J}^{14}$.

\section{Teste de incapacidade funcional (Tempo de elevação da pata)}

No teste descrito originalmente por Tonussi e Ferreira ${ }^{15}$, os animais deambularam sobre um cilindro metálico, de aproximadamente $30 \mathrm{~cm}$ de diâmetro, recoberto por tela de aço inoxidável, que por meio de um motor elétrico realizava 3 rotações por minuto. Nos membros posteriores dos animais, foram adaptadas botas metálicas que conduziam a informação da pata direita, por meio de um fio, a um computador contendo um programa que mostrava os valores de tempo no qual a pata do animal manteve-se no ar, enquanto o mesmo permaneceu deambulando sobre o cilindro, em um minuto. 
O experimento iniciou com o treino dos animais e, no dia seguinte, foram anotados os valores de tempo da marcha normal (pré-lesão). Em seguida, houve o procedimento traumático, sendo que as reavaliações ocorreram: uma hora após a indução da lesão, ou seja, pós-lesão; após o primeiro dia de tratamento ou sham, isto é, no mesmo dia em que ocorreu a lesão; as outras reavaliações foram realizadas após 2,8 e 24 horas da lesão (antes de ocorrer a segunda terapia); e finalmente após o $5^{\circ}$ dia de lesão. Normalmente, os animais sem alterações exibem, em sua marcha, a manutenção da pata no ar em torno de dez segundos, já os animais com dor apresentam maiores tempos ${ }^{14}$.

\section{Avaliação do edema}

Avaliou-se o diâmetro dos tendões, em seu terço médio, com paquímetro posicionado médio-lateralmente. Esta forma de avaliação foi realizada após o teste de incapacidade funcional, nos mesmos períodos ${ }^{14}$.

\section{Protocolos de tratamento}

Após a avaliação do momento pós-lesão, iniciou-se o tratamento, utilizando o ultrassom (Ibramed ${ }^{\circledR}$ ), com frequência de $1 \mathrm{MHz}, 1 \mathrm{~cm}^{2} \mathrm{ERA}$, de forma contínua ou pulsada $(100 \mathrm{~Hz})$, durante 3 minutos, sobre o local do trauma, acoplamento com gel, realizando movimentos circulares lentos ${ }^{16}$; os animais foram mantidos em contensor de PVC. O grupo GS sofreu procedimento semelhante, porém com o equipamento desligado.

De acordo com a literatura, a técnica usada foi não térmica ${ }^{17}$, sendo utilizados $0,4 \mathrm{~W} / \mathrm{cm}^{2}$ para GUC e 2,0 W/ $\mathrm{cm}^{2}$ de SATP para GUP; como foi utilizado um ciclo de trabalho de $20 \%$, a dose média temporal era também de $0,4 \mathrm{~W} / \mathrm{cm}^{2}$ (SATA). O equipamento possuía certificado de calibração válido durante o experimento.

O procedimento ocorreu no decorrer de 5 dias consecutivos, a contar o dia da lesão, num total de 5 terapias, com intervalo de 24 horas. Ao final do experimento, os animais sofreram eutanásia por decapitação em guilhotina.

\section{Análise dos resultados}

Os resultados foram avaliados quanto a sua normalidade, por meio do teste de Kolomogorv-Smirnov; expressos e analisados por meio de média, desvio padrão, análise de variância com medidas repetidas e pós-teste de Tukey, com nível de significância de $\alpha=0,05$.

\section{RESULTADOS}

\section{Tempo de elevação da pata}

Para GS, comparando com o momento pré-lesão $(\mathrm{p}<0,001)$, houve aumento significativo dos tempos. Ao realizar as comparações entre os valores após lesão e os seguintes, não foram encontradas variações significativas $(p>0,05)$.

Para GUC, ao comparar o momento pré-lesão com os momentos seguintes, observou-se aumento significativo até 8 horas $(\mathrm{p}<0,001)$, mas a partir de 24 horas tal variação não foi mais significativa ( $p>0,05)$. Nas comparações com os valores pós-lesão, houve diminuição significativa com o tratamento a partir de 24 horas $(\mathrm{p}<0,05)$.

Para GUP, as comparações com os valores pré-lesão apresentaram aumento significativo apenas até 2 horas $(p<0,001)$, não ocorrendo diferenças a partir de 8 horas $(p>0,05)$. Ao comparar com pós-lesão, houve diminuição significativa a partir de 8 horas $(\mathrm{p}<0,01)$ e ao comparar com a última avaliação, $\mathrm{p}<0,05$ (Figura 1).

$\mathrm{Na}$ comparação entre os grupos, apenas em 8 horas houve diferença significativa entre GUC e GUP, sendo os valores de GUC significativamente maiores $(\mathrm{p}<0,01)$.

\section{Avaliação do edema}

Para GS, ao comparar o momento pré-lesão com os momentos seguintes, houve aumento significativo dos diâmetros $(\mathrm{p}<0,001)$ - comparativamente com a última avaliação $(p<0,01)$. Ao realizar as comparações entre os valores após a lesão, com os dos momentos seguintes, foi encontrada redução significativa apenas 24 horas após a lesão $(\mathrm{p}<0,05)$ e no $5^{\circ}$ dia $(\mathrm{p}<0,001)$.

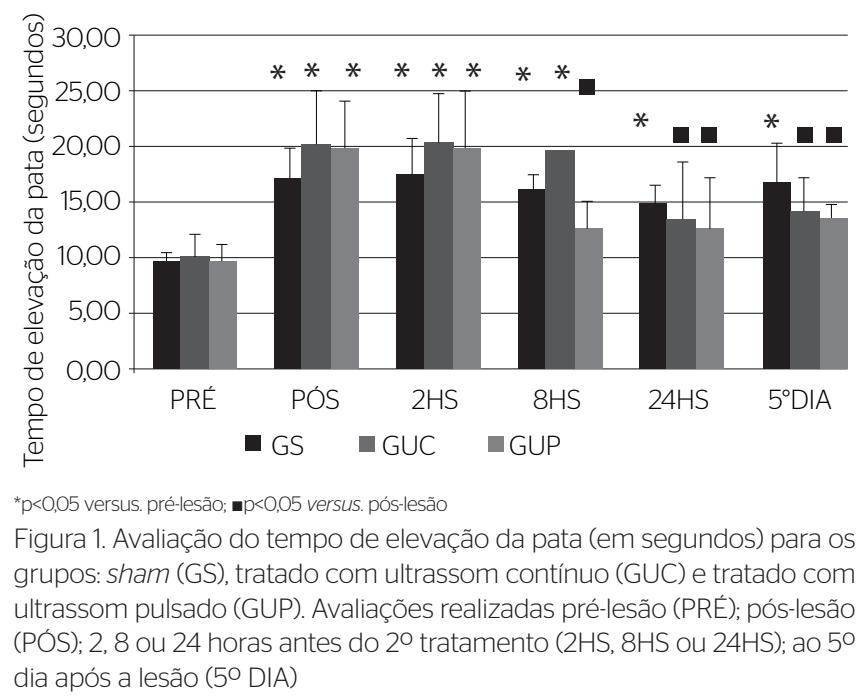


GUC e GUP se comportaram de forma semelhante, ao comparar o momento pré-lesão com os momentos seguintes houve aumento significativo até 8 horas ( $\mathrm{p}<0,001)$. Nas comparações com os valores pós-lesão, houve variação significativa em todos os momentos, sendo que os valores após 2 horas $(\mathrm{p}<0,001)$ e 8 horas $(\mathrm{p}<0,01)$ foram maiores, e os valores após 24 horas e no $5^{\circ}$ dia $(\mathrm{p}<0,001)$ manifestaram-se menores (Figura 2$)$.

$\mathrm{Na}$ comparação entre os grupos houve diferenças desde a primeira avaliação, entre GUC e GUP, sendo que os valores do primeiro foram maiores $(p<0,05)$. $\mathrm{Na}$ avaliação após a lesão, a diferença foi observada entre GS e GUP (menor para GUP) (p<0,01). Em 2 e 8 horas, os valores de GS foram menores tanto quando comparados com GUC $(p<0,05)$ quanto com GUP ( $<<0,01)$. Já para 24 horas e $5^{\circ}$ dia, as diferenças se inverteram, com maiores valores de GS quando comparados com GUC e GUP $(\mathrm{p}<0,01)$.

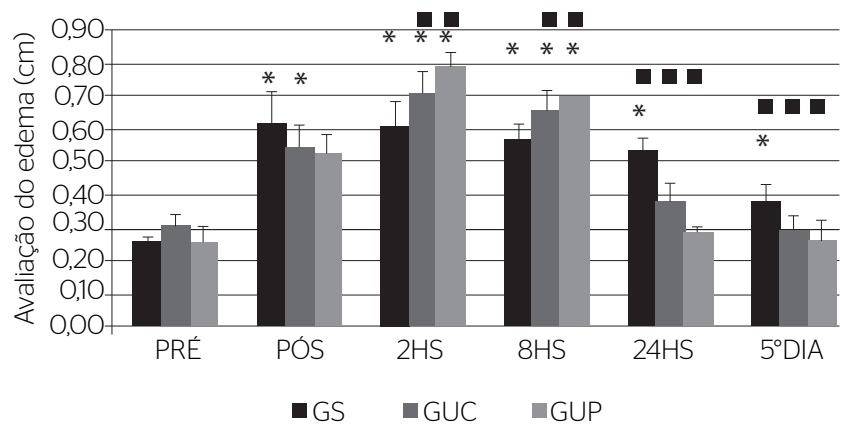

${ }^{*} \mathrm{p}<0,05$ versus. pré-lesão; $\mathbf{m}<0,05$ versus. pós-lesão

Figura 2. Avaliação do edema no tendão calcâneo (diâmetro em centímetros) para os grupos: sham (GS), tratado com ultrassom contínuo (GUC) e tratado com ultrassom pulsado (GUP). Avaliações realizadas pré-lesão (PRÉ); pós-lesão (PÓS); 2, 8 ou 24 horas antes do 20 tratamento (2HS, 8HS ou 24HS); ao 50 dia após a lesão (50 DIA).

\section{DISCUSSÃO}

Diversos modelos experimentais são usados para avaliar as doenças e o reparo dos tendões, como injeção de carragenina e prostaglandina $\mathrm{E}_{2}\left(\mathrm{PGE}_{2}\right)^{4}$; injeção de colagenase ${ }^{6,18}$; ruptura parcial longitudinal ${ }^{19}$; secção transversa parcial ${ }^{11,12}$; secção transversa total ${ }^{9}$; e lesões agudas diretas por contusão ${ }^{20}$.

A inflamação peritendínea pode se transformar em doença intratendínea, o que pode favorecer a degradação do tendão. Desta forma, o tratamento anti-inflamatório previne as alterações estruturais progressivas $^{4}$. Assim, salienta-se a importância de estudos que avaliem processos inflamatórios na região peritendínea com modalidades terapêuticas, como realizado no presente estudo, tendo o trauma direto como modelo, e avaliando-se dois sinais do processo inflamatório (dor e edema), sendo que ambos foram constatados.

A avaliação da dor foi realizada por meio do tempo de elevação da pata $^{15}$, que avalia a capacidade do animal em deambular com adequada descarga de peso no membro posterior, visto que após o trauma os animais apresentam dor no tendão, tal fato os levou a aumentar o tempo em que permaneciam com a pata no ar (TEP). Para o grupo controle, tal fato foi observado durante todo o período experimental.

De acordo com Warden e McMeeken ${ }^{13}$, o ultrassom é um instrumento frequentemente usado na fisioterapia esportiva, apesar de lacunas sobre seus efeitos clínicos e falta de consenso dosimétrico, significando que são necessárias mais pesquisas que o avaliem, variando-se a forma de entrega, mas com dose média espacial e temporal semelhantes.

Assim, foi possível observar efeitos positivos com relação à nocicepção, analisada pelo TEP. Nas duas formas de tratamento houve diminuição nos valores de TEP após a lesão, com restauração dos valores iniciais em ambos os grupos. Tal fato é corroborado pelos dados de Madiman, Wasel e Fisher ${ }^{21}$ que, avaliando o limiar de dor à pressão em humanos, relataram que o ultrassom de 1,1 MHz, com dose de 1,0 W/ $\mathrm{cm}^{2}$, produziu aumento no limiar. Kozanoglu et al. ${ }^{22}$ relatam melhora da dor, em pacientes com osteoartrite de joelho, com o uso do ultrassom de $1 \mathrm{MHz}$, com $1 \mathrm{~W} / \mathrm{cm}^{2}$, de forma contínua. Ebenbichler et al. ${ }^{23}$ e Bakhtiary e RashidyPour ${ }^{24}$ relataram alívio da dor, em pacientes com síndrome do túnel do carpo, usando ultrassom de $1 \mathrm{MHz}$, com $1 \mathrm{~W} / \mathrm{cm}^{2}$, pulsado $20 \%$. Já Coertjens et al. ${ }^{25}$, utilizando equipamento de $3 \mathrm{MHz}$ e dose $0,2 \mathrm{~W} / \mathrm{cm}^{2}$, pulsado $20 \%$, relatam que apesar de observarem diminuição da dor, em pacientes cirúrgicos cardiovasculares, o resultado não foi significativamente diferente do observado para o grupo placebo. Fato semelhante foi observado por D'Vaz et al. ${ }^{26}$ ao usar ultrassom de baixa intensidade $\left(30 \mathrm{~mW} / \mathrm{cm}^{2}, 1,5 \mathrm{MHz}\right)$ em pacientes com epicondilite lateral crônica.

Para o edema também foi possível vislumbrar efeitos benéficos do ultrassom, após 24 horas, pois em períodos anteriores o ultrassom promoveu aumento do edema, o que pode ter ocorrido por uma possível ação pró-inflamatória. De forma contrária, $\mathrm{Fyfe}^{27}$, avaliando a formação de edema por injeção de nitrato de prata, utilizou 3 frequências $(3,1,5$ e 0,75 MHz) em duas formas de pulsado 
(20 e 50\%), na dose de $0,5 \mathrm{~W} / \mathrm{cm}^{2}$, e observou que para $0,75 \mathrm{MHz}$ houve redução significativa do edema após 30 minutos da indução. Por outro lado, Franco et al. ${ }^{28}$ não observaram nenhum efeito do uso do ultrassom $3 \mathrm{MHz}$, com dose de $0,3 \mathrm{~W} / \mathrm{cm}^{2}$, sobre o edema em patas de ratos, induzido por injeção de carragenina.

Contudo, vale salientar que nas primeiras reavaliações (duas e oito horas), após o tratamento inicial, para o TEP, apesar de não ocorrer diferenças entre os grupos tratados e o sham, houve ligeiro aumento para GUC e GUP em duas horas e diferença significativa entre os dois grupos em oito horas, com diminuição da nocicepção apenas para o grupo tratado com ultrassom pulsado. Para o edema, os grupos tratados tiveram aumentos significativos em relação ao que foi observado para o grupo sham. Segundo Watson ${ }^{17}$, o ultrassom terapêutico pode aumentar o fluxo sanguíneo local e - como produz efeito estimulante sobre mastócitos, plaquetas e leucócitos em geral (podendo causar a degranulação de mastócitos e consequente aumento inicial do processo inflamatório) - piorar o quadro inicial de uma lesão. Tal fato pode ter ocorrido no presente estudo, mas visto que Watson ${ }^{17}$ cita que o ultrassom é um otimizador do processo inflamatório, em 24 horas os efeitos positivos puderam ser observados, sendo que para GUP, quanto ao TEP, os resultados já iniciaram em oito horas.

O uso do ultrassom sobre o reparo de tenotomias é objeto de estudo de diversos autores, tais como Yeung, Guo e $\mathrm{Ng}^{12}$, que relataram o uso de $1 \mathrm{MHz}, 2,5 \mathrm{~W} / \mathrm{cm}^{2}$, $20 \%$ (SATA de $0,5 \mathrm{~W} / \mathrm{cm}^{2}$ ), por cinco minutos, durante duas semanas, produzindo melhor alinhamento do colágeno e tendões mais fortes do que o controle. Cunha, Parizotto e Vidal ${ }^{9}$, com protocolo semelhante, observaram sob microscopia de luz polarizada, maior birrefringência para os tendões que utilizaram o modo pulsado, mostrando melhor organização e agregação do colágeno.

$\mathrm{Ng}$ et al. ${ }^{10}$ utilizaram ultrassom contínuo e, avaliando com ensaio de carga, encontraram uma maior força de ruptura para o grupo que usou $2,0 \mathrm{~W} / \mathrm{cm}^{2}$ do que para $1,0 \mathrm{~W} / \mathrm{cm}^{2}$. Achado que foi reproduzido ao comparar, além das doses citadas, natação e corrida em estei$\mathrm{ra}^{11}$. Contudo, posteriormente, $\mathrm{Ng}$ e Fung ${ }^{29}$ avaliaram a morfologia da cura do tendão, com grupos de 0, 0,5,1,2 e $2,0 \mathrm{~W} / \mathrm{cm}^{2}$; e encontraram que o tamanho das fibrilas de colágeno era maior para os grupos tratados, com uma tendência a diminuir o diâmetro médio das fibrilas com o aumento da intensidade.

De acordo com Watson ${ }^{17}$, para que ocorram efeitos terapêuticos térmicos, a temperatura tecidual deve aumentar entre $40-45^{\circ} \mathrm{C}$, durante ao menos 5 minutos.
Assim, no presente estudo, mesmo levando-se em conta a limitação de falta de controle do aumento de temperatura intratendíneo, acredita-se que não houve hipertermia terapêutica, o que segundo Watson ${ }^{17}$ não significa que não tenha ocorrido aumento de temperatura local.

Vislumbrou-se que os melhores resultados pertenceram ao GUP, que retornaram aos valores iniciais mais rapidamente do que no GUC. Apesar dos dois possuírem doses temporais médias iguais, vale ressaltar que para GUP a dose temporal de pico foi cinco vezes superior. Os efeitos terapêuticos do ultrassom não térmico advêm da cavitação estável e do microfluxo acústico, que podem alterar a estrutura, permeabilidade e função da membrana celular, estimulando a rápida resolução do processo inflamatório, o reparo e regeneração tecidual ${ }^{30}$, e assim produzindo efeitos analgésicos e de redução do edema.

Salienta-se, como limitação do estudo, a falta de correlação com aspectos morfológicos da lesão. Sendo sugestão para futuros estudos, além do uso de doses térmicas e outras frequências do ultrassom.

\section{CONCLUSÃO}

O ultrassom terapêutico produziu diminuição de dor e de edema, em animais com tendão calcâneo traumatizado, sendo que tal ação foi mais precoce para o ultrassom na forma pulsada.

\section{REFERÊNCIAS}

1. Sandrey MA. Acute and chronic tendon injuries: factors affecting the healing response and treatment. J Sport Rehabil. 2003;12(1):70-91.

2. Archambault J. Tendon micromechanics and research methods in tendinopathy. J Musculoskelet Neuronal Interact. 2003;3(4):326-8.

3. Vogel KG. Tendon structure and response to changing mechanical Ioad. J Musculoskelet Neuronal Interact. 2003;3(4):323-5.

4. Aspenberg P. Is inflammation harmless to loaded tendons? J Appl Physiol. 2007;102(1):3-4.

5. Woo SLY, Debski RE, Zeminski J, Abramowitch SD, Saw SSC, Fenwick JA. Injury and repair of ligaments and tendons. Annu Rev Biomed Eng. 2000;2:83-118.

6. Marsolais D, Côté CH, Frenette J. Nonsteroidal anti-inflammatory drug reduces neutrophil and macrophage accumulation but does not improve tendon regeneration. Lab Invest. 2003,83(7):991-9.

7. Koeke PU, Parizotto NA, Carrinho PM, Salate ACB. Comparative study of the efficacy of the topical application of hydrocortisone, therapeutic ultrasound and phonophoresis on the tissue repair process in rat tendons. Ultrasound Med Biol. 2005;31(3):345-50. 
8. Ng GYF, Wong RYF. Ultrasound phonophoresis of panax notoginseng improves the strength of repairing ligament: a rat model. Ultrasound Med Biol. 2008;34(12):1919-23.

9. Cunha A, Parizotto NA, Vidal BC. The effect of therapeutic ultrasound on repair of the achilles tendon (tendo calcaneus) of the rat. Ultrasound Med Biol. 2001;27(12):1691-6.

10. Ng COY, Ng GYF, See EKN, Leung MCP. Therapeutic ultrasound improves strength of Achilles tendon repair in rats. Ultrasound Med Biol. 2003;29(10):1501-6

11. Ng GYF, Ng COY, See EKN. Comparison of therapeutic ultrasound and exercises for augmenting tendon healing in rats. Ultrasound Med Biol. 2004;30(11):1539-43

12. Yeung CK, Guo X, Ng YF. Pulsed ultrasound treatment accelerates the repair of Achilles tendon rupture in rats. J Orthop Res. 2006:24(2):193-201.

13. Warden SJ, Mcmeeken JM. Ultrasound usage and dosage in sports physiotherapy. Ultrasound Med Biol. 2002;28(8):1075-80.

14. Bertolini GRF, Silva TS, Ciena AP, Trindade DL. Efeitos do laser de baixa potência sobre a dor e edema no trauma tendíneo de ratos. Rev Bras Med Esporte. 2008;14(4):362-6.

15. Tonussi CR, Ferreira SH. Rat knee-joint carrageenin incapacitation test: an objective screen for central and peripheral analgesics. Pain. 1992;48(3):421-7.

16. Demmink JH, Helders PJM. The effect of moving an ultrasound transducer on tissue heating. Adv Physiother. 2003;5(2):50-6.

17. Watson T. Ultrasound in contemporary physiotherapy practice. Ultrasonics. 2008;48(4)321-9.

18. Dahlgren LA, Mohammed HO, Nixon AJ. Expression of insulin-like growth factor binding proteins in healing tendon lesions. J Orthop Res. 2006;24(2):183-92.

19. Demir H, Menku P, Kirnap M, Calis M, Ikizceli I. Comparison of the effects of laser, ultrasound, and combined laser + ultrasound treatments in experimental tendon healing. Lasers Surg Med. 2004;35(1):84-9.
20. Lin TW, Cardenas L, Soslowsky LJ. Biomechanics of tendon injury and repair. J Biomech. 2004;37(6):865-77

21. Madiman S, Weasel J, Fisher B. The effect of ultrasound on the mechanical pain threshold of healthy subjects. Physiotherapy. 1995;81(12):718-23.

22. Kozanoglu E, Basaran S, Guzel R, Guler-Uysal F. Short term efficacy of ibuprofen phonophoresis versus continuous ultrasound therapy in knee osteoarthritis. Swiss Med Wkly. 2003;133(23-24): 333-8.

23. Ebenbichler GR, Resch KL, Nicolakis P, Wiesinger GF, Uhl F, Ghanem $\mathrm{AH}$, et al. Ultrasound treatment for treating the carpal tunnel syndrome: randomised "sham" controlled trial. BMJ. 1998;316; 731-5.

24. Bakhtiary AH, Rashidy-Pour A. Ultrasound and laser therapy in the treatment of carpal tunnel syndrome. Aust J Physiother. 2004:50(3):147-51.

25. Coertjens PC, Coertjens M, Bernardes C, Prati FA, Sá SLR. Avaliação dos níveis de dor após sessões de ultra-sonoterapia em pacientes cirúrgicos cardiovasculares. Rev Bras Fisioter. 2005;9(1):25-31.

26. D'Vaz AP, Ostor AJK, Speed CA, Jenner JR, Bradley M, Prevost AT, et al. Pulsed low-intensity ultrasound therapy for chronic lateral epicondylitis: a randomized controlled Trial. Rheumatology. 2006:45(5):566-70

27. Fyfe MC. A study of the effects of different ultrasonic frequencies on experimental oedema. Aust J Physiother. 1979;25(5):205-7.

28. Franco AD, Pereira LE, Groschitz M, Aimbire F, Martins RABL, Carvalho RA. Análise do efeito do ultra-som no edema inflamatório agudo estudo experimental. Fisioter Mov. 2005;18(2):19-24.

29. Ng GYF, Fung DTC. The effect of therapeutic ultrasound intensity on the ultrastructural morphology of tendon repair. Ultrasound Med Biol. 2007:33(11):1750-4

30. Speed CA. Therapeutic ultrasound in soft tissue lesions. Rheumatology. 2001:40(12):1331-6 